\title{
Social Distance Analysing - COVID-19 Using Deep Learning and Computer Vision
}

\author{
Kishan Ghanshyam Poriya ${ }^{1}$, Prof. Surabhi Thorat ${ }^{2}$, Prof. Swati Maurya ${ }^{3}$ \\ ${ }^{1}$ Student, Department of Computer Science, S.K.Somaiya College, Somaiya Vidyavihar University, Mumbai, \\ India \\ ${ }^{2,3}$ Professor, Department of Computer Science, S.K.Somaiya College, Somaiya Vidyavihar University, Mumbai, \\ India
}

\begin{abstract}
Article Info

Volume 7, Issue 6

Page Number: 196-202

Publication Issue :

November-December-2021

\section{Article History}

Accepted : 02 Dec 2021

Published : 10 Dec 2021

In the combat in opposition to the coronavirus, social distancing has tested to be an effective degree to bog down the unfold of the disease. The machine provided is for reading social distancing through calculating the space among humans for you to gradual down the unfold of the virus. This machine makes use of enter from video frames to parent out the space among people to relieve the impact of this pandemic. This is performed through comparing a video feed acquired through a surveillance camera. The video is calibrated into bird's view and fed as an enter to the YOLOv3 version that is an already educated item detection version. The YOLOv3 version is educated using the Common Object in Context (COCO). The proposed machine turned into corroborated on a pre-filmed video. The outcomes and consequences acquired through the machine display that assessment of the space among more than one people and figuring out if policies are violated or not. If the space is less than the minimal threshold value, the people are represented through a purple bounding box, if not then it's far represented through a inexperienced bounding box. This machine may be similarly advanced to detect social distancing in real-time applications.
\end{abstract}

Keywords : Social distancing. Object detection. Crowd analysis, covid-19

\section{INTRODUCTION}

A recent study indicates that social distancing is an important and essential containment measure to prevent SARSCoV2, because people with mild or absent symptoms can be carriers of a corona infection at random and can infect other people. In the below figure indicates that adequate social distancing is the best way to reduce infections through physical contact, thereby reducing the rate of infection. This reduced spike can certainly match the available healthcare infrastructure and help provide better facilities for patients battling the coronavirus pandemic. 
Maintaining social distancing in public spaces such as transit stations, shopping malls, and college campuses is key to preventing or slowing the spread of the virus. The practice of social distancing (SD) could continue in the following years until the spread of the virus is completely eliminated.

This article aims to mitigate the effects of coronavirus disease with minimal loss of resources; this disease has had a serious impact on the world economy. Second, provide a highly accurate people detection solution that helps monitor social distancing at night. Especially, in the summer when the heat is at its peak, people with crowded homes find themselves outside their homes at night with their families for some fresh air. During this serious situation, appropriate measures should be taken. Recently, Eksin et al.evaluated the SIR (Infected Susceptible Healed) model in which they included a social distance term. They have shown that the spread of the disease depends on the social behavior of people. They evaluated the results of the SIR model with and without the behavior change factor and found that a simple SIR model did not perform well even after many repeated observations; while their updated SIR model with a behavior change factor showed good results and corrected the initial error rate. In a similar context, a disembarkation AI company said the development of an AI tool to monitor social distance in the work area.In a brief report, the company said the potential tool would be able to observe people, whether or not they meet the safety distance criteria, by examining real-time video feeds captured by the camera. They said this tool can be easily combined with the security cameras available in different functional areas to ensure a safe distance between workers. The world's leading research firm, Gartner Inc., said AI has become an attractive provider of core AI technologies to recognize their timely push to support the fight against the deadly COVID19 situation.

\section{RELATED WORK}

This section introduces and highlights some work related to object detection and people detection using deep learning. A large number of recent articles dealing with the classification and detection of objects involving deep learning are also discussed. Human detection performed using computer vision is considered part of object detection. Detected objects are located and classified according to their shape using a predefined model. Techniques using convolutional neural networks $(\mathrm{CNN})$ and deep learning have been shown to perform better on visual recognition cues. and drawbacks such as speed and precision. Real-time object detection algorithms using the CNN model such as Regional Convolutional Neural Networks (RCNN) and You Only Look Once (YOLO) are developed for the detection of multiple classes in various regions. YOLO (You Only Look Once) is a cutting edge technique for speed and accuracy in CNN based object detection.

By transforming the objective and interpretation from the work, this proposed system presents a method of detecting people using computer vision. Instead of using drone technology, the input is a stream of video footage from an installed CCTV camera. The camera's field of view covers pedestrians passing within range of the installed camera. People in the photo are shown using a bounding box using deep CNN patterns. CNN-based deep YOLO algorithm is used to detect people in the video stream sequence filmed by the CCTV camera. The calculations are done by measuring the barycentric distance between pedestrians, this will represent whether the pedestrians in the video follow sufficient social distance

Any researcher working in the medical and pharmaceutical fields aiming at the treatment of the infectious disease COVID19; however, a definitive solution has not yet been found. On the other hand, 
controlling the spread of such an unknown infectious respiratory disease is another issue.

Convolutional Neural Networks (CNNs) have played a very important role in feature extraction and classification of complex objects. By developing faster CPUs, GPUs, and expanded memory capacities, CNNs allow researchers to build detectors that are accurate and faster than conventional models. training, detection speed and achieving better accuracy are still challenges to be solved.

\section{METHODOLOGY}

We advise a 3-degree version such as human beings detection, tracking, inter-distance estimation as a complete answer for social distancing tracking and zone-primarily based totally contamination chance analysis. The gadget may be incorporated and carried out on all kind of CCTV surveillance cameras with any decision from VGA to Full-HD, with real-time performance.

\section{DESIGN METHODOLOGY}

In design methodology we discuss about the following points

- Collection of inputs

- Pedestrians

- Python

- Distance measurment

\section{Collection of inputs}

The video recorded by the CCTV camera is given as the input. The camera is set up in a way that it captures at a fixed angle. The frame will change into a $2 \mathrm{D}$ view to accurately estimate the distance between people.
The distance between peoples is easily estimated and measured by calculating the distance between the centroids. A present minimum value for the distance is set already.It is depending upon this value, any distance lower than the present minimum distance value which we set already is found, then a warning is shown using red colored bounded box. If the distance is safe distance then the bounded box will turn it green, if the distance between the people is in medium distance which is not in minimal distance and not the maximum distance then the bounded box will be turn to orange

\section{Pedestrians}

Deep Convolutional neural networks model is a simple and efficient model for object detection. This model considers the region which contain only "Person". The object detection approach used in the social distancing analyzer model reduces the computational complexity issues. It is done by formulating the detection of objects with the help of a single regression problem. In object detection models based on deep learning, the You Only Look Once model. This model is suitable for real-time applications and it is faster and provides accurate results.

YOLOv3 is that the latest variant of we tend tollliked|a preferred\} object detection algorithmic program YOLO - you simply Look Once. The revealed model acknowledges eighty completely different objects in pictures and videos, however most significantly it's super-fast and nearly as correct as Single Shot MultiBox (SSD). First, it divides the image into a $13 \times 13$ grid of cells. the dimensions of those 169 cells varies counting on the size of the input. For a $416 \times 416$ input size that we utilized in our experiments, the cell size was $32 \times 32$. every cell is then to blame for predicting variety of boxes within the image. for every bounding box, the network additionally predicts the boldness that the bounding box really encloses associate degree object, and 
therefore the chance of the basined object being a specific class. Most of those bounding boxes are eliminated as a result of their confidence is low or because they're enclosure an equivalent object as another bounding box with terribly high confidence score. this method is named non most suppression.

In Below Figure suggests the pedestrian detection the usage of the YOLOv3 version. The YOLOv3 is an item detection version that takes an photo or a video as an enter and may concurrently research and draw bounding field coordinates (tx, ty, tw, th), corresponding magnificence label probabilities ( $\mathrm{P} 1$ to Pc), and item confidence. The YOLOv3 is an already educated version at the Common Objects in Context dataset (COCO dataset). This dataset includes eighty labels along with a human magnificence called pedestrian magnificence. The Figure five represents the YOLOv3 version utilized in Social Distancing Analyzer, The parameters used withinside the detection of pedestrians are as follows:

- Box Coordinates - tx, ty, tw, th

- Object Confidence - C

- Pedestrians - P1, P2, ... Pc

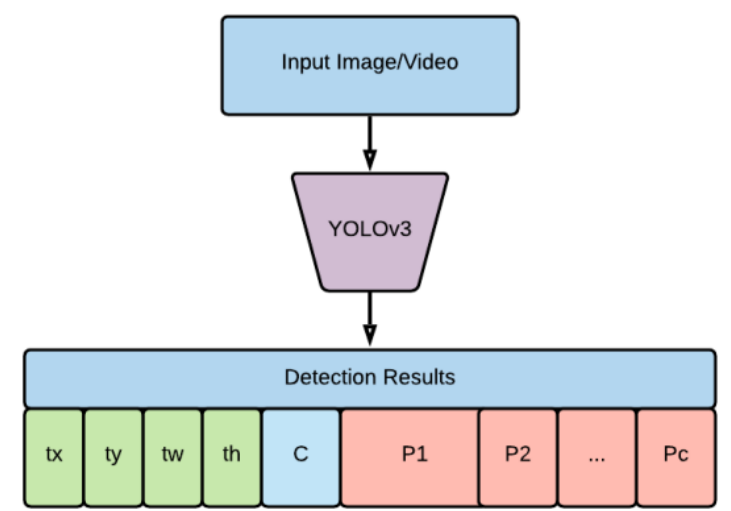

Figure: Detection of pedestrians using YOLOv3

There are several objects present in the same frame, the goal is to identify the bounding boxes of the "Person Only" class map relating to people only. The code for drawing the bounding boxes is shown below and the output of this code is shown in the figure below.

$$
\begin{aligned}
& \text { \#To identity "Person Only" class } \\
& \mathrm{x}=\mathrm{np} \cdot \text { where(classes==0)[0] } \\
& \mathrm{p}=\mathrm{box}[\mathrm{x}] \\
& \operatorname{count}=\operatorname{len}(\mathrm{p}) \\
& \mathrm{x} 1, \mathrm{y} 1, \mathrm{x} 2, \mathrm{y} 2=\mathrm{p}[0] \\
& \operatorname{print}(\mathrm{x} 1, \mathrm{y} 1, \mathrm{x} 2, \mathrm{y} 2)
\end{aligned}
$$

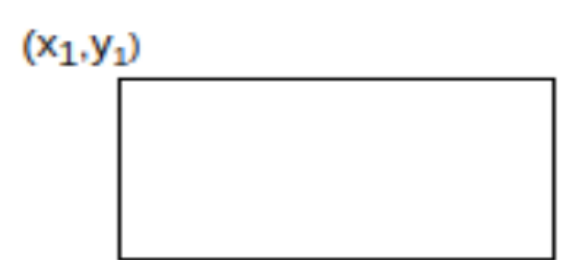

$\left(\mathrm{x}_{2}, \mathrm{y}_{2}\right)$

Figure : Output Obtained from Bounded Box Method

\section{Python :-}

Python is a deciphered, significant level, broadly useful programming language. Made by Guido van Rossum and first delivered in 1991, Python's plan theory stresses code coherence with its outstanding utilization of critical whitespace. Its language develops and object-situated methodology plan to assist software engineers with composing clear, intelligent code for little and largescale projects.

Python is powerfully composed and garbage collected. Python is frequently depicted a language because of its thorough standard library. Python utilizes dynamic composing and a blend of reference counting and a cycle recognizing garbage man for memory the board. It likewise includes dynamic name goal, which ties strategy and variable names during program execution.

\section{Distance Measurement}

The stretch between the arrangement of individuals in partner degree input outline are regularly basically determined once the jumping box for each individual is planned. to attempt to do accordingly the base focal 
point of the case planned to every individual inside the differ is thought of.In Figure addresses the means followed by the social removing instrument model in order to compute the hole and create alerts.

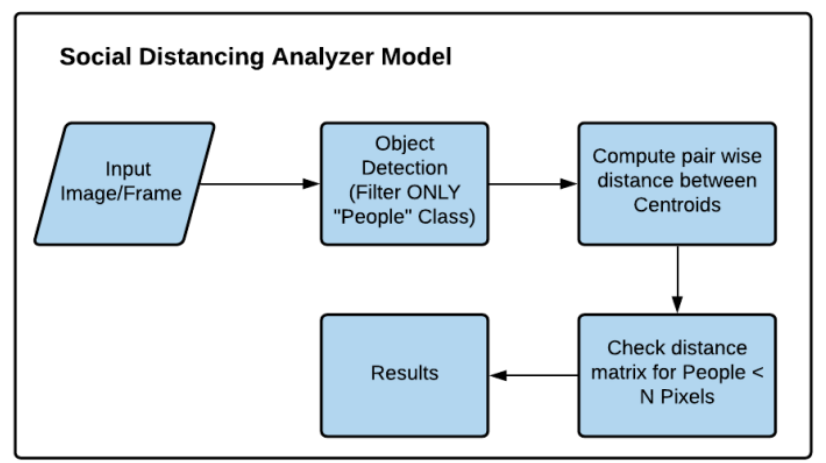

Figure : Social distance analyzer model which involve the steps

For every individual in the information outline, the direction in the bird's view change is determined dependent on the focal hub point of each individual in the info outline. The distance timespan set of individuals can be assessed from the bird's view by ascertaining the euclidean distance between centroids. As the camera is aligned, more precise outcomes can be acquired. The arrangement of people whose span is lower than the preset least limit esteem is considered as infringement. Individuals who disregard the condition are stamped utilizing a red box, and the leftover individuals are checked utilizing a green box. The code for figuring the focuses of the cases of are given below:

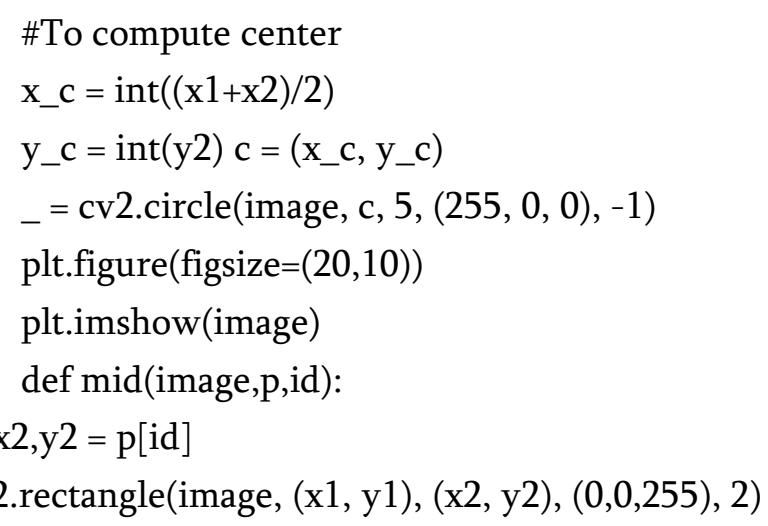

_ = cv2.rectangle(image, $(\mathrm{x} 1, \mathrm{y} 1),(\mathrm{x} 2, \mathrm{y} 2),(0,0,255), 2)$

$\mathrm{x} 1, \mathrm{y} 1, \mathrm{x} 2, \mathrm{y} 2=\mathrm{p}[\mathrm{id}]$
The code to compute the pairwise distances between all detected people in a frame is given below:

$\% \%$ time from scipy.spatial import distance def dist(midpt,n):

$\mathrm{d}=\mathrm{np} \cdot \operatorname{zeros}((\mathrm{n}, \mathrm{n}))$

for $i$ in range $(n)$ :

for $\mathrm{j}$ in range $(\mathrm{i}+1, \mathrm{n})$ :

if $\mathrm{i} !=\mathrm{j}$ :

dst $=$ distance.euclidean $(\operatorname{midpt}[\mathrm{i}], \operatorname{midpt}[\mathrm{j}])$

$\mathrm{d}[\mathrm{i}][\mathrm{j}]=\mathrm{dst}$

return $\mathrm{d}$

If the end result acquired withinside the preceding technique is much less than the minimal appropriate threshold value, then the field across the set of humans is represented the use of red colour. The code that defines a feature to alternate the colour of the nearest humans to red is given below:

def red(image,p,p1,p2):

unsafe $=$ np.unique $(p 1+p 2)$

for $\mathrm{i}$ in unsafe:

$\mathrm{x} 1, \mathrm{y} 1, \mathrm{x} 2, \mathrm{y} 2=\mathrm{p}[\mathrm{i}]$

${ }_{-}=$cv2.rectangle(image, $\left.(\mathrm{x} 1, \mathrm{y} 1),(\mathrm{x} 2, \mathrm{y} 2),(255,0,0), 2\right)$

return image

\section{Result And Discussion}

The video indicates the pedestrian strolling on a public street. In this work, the video body is constant at a unique attitude to the street. The attitude view of the video body is converted right into a pinnacledown view for a extra correct estimation of distance measurement. indicates the social distancing detection in a video body and the consequences of the pinnacle-down view. The sequences are depicted from pinnacle to bottom. The factors constitute every pedestrian for social distancing detection.

The red points represent the pedestrians whose distance with another pedestrian is below the acceptable threshold and the green points represent 
the pedestrians who keep a safe distance from other pedestrians. However, there are also some detection errors shown. These errors are possibly due to the pedestrians walking too near to another pedestrian until they are overlaid on the camera view. The precision of the distance measurement between pedestrians is also affected by the pedestrian detection algorithm.

The YOLO algorithmic program is additionally able to find the 0.5 body of the pedestrian as associate degree object by showing the bounding box, the position of the pedestrian admire the middle-point of very cheap line is calculable supported the bounding box are going to be less precise. to beat the detection errors, the planned methodology had been improved by adding a quadrilateral box to watch the appointed region in a picture as shown in. Hence, solely the pedestrians walking inside the desired area will be counted for folks density measurement.

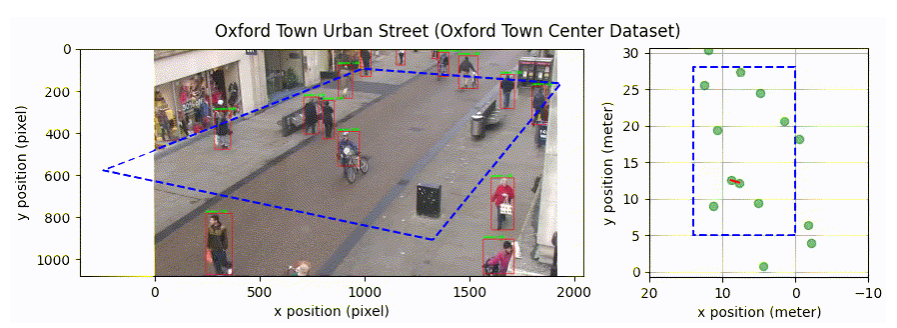

\section{CONCLUSION AND FUTURE WORK}

A social distance analysis tool is proposed. The system uses computer vision and a deep learning model. With the help of computer vision, the distance between each person can be easily calculated. If it turns out that a set of individuals does not meet the minimum accepted threshold value, this will be indicated by a red bounding box. The developed system uses pre-filmed video of people on a crowded street.
The number of violations is confirmedby calculating the number of groups formed and the term of the violation index calculated as the ratio between the number of people and the number of groups. Extensive studies were conducted with state-of-theart object detection models: FasterRCNN, SSD and YOLO v3, where YOLO v3 demonstrated effective performance with balanced FPS and mAP score. Since this approach is very sensitive to the spatial position of the camera, the same approach can be refined to better match the corresponding field of view.

The proposed system is able to estimate the distance between people. Social distancing patterns are distinct and categorized into "safe" and "unsafe" distance. Moreover, it also displays labels based on object detection and classification. The categorizer can be implemented for live video streams and can be used for real-time application development. This system can be integrated with video surveillance for monitoring people during pandemics. Mass screening is feasible and therefore is often used in crowded places such as train stations, bus stops, markets, streets, entrances to shopping malls, schools, colleges, workplaces and restaurants. By monitoring the space between two individuals, we can confirm that a safe distance is maintained, this can help us curb the virus.

\section{REFERENCES}

[1]. COVID19.who.int. (n.d.). WHO coronavirus disease (COVID-19) dashboard. Available at: https://covid19.who.int [Accessed 1 Jul 2020]

[2]. Advice for the public on COVID-19-World Health Organization. WHO www.who.int/emergencies/diseases/novelcoronaviru s-2019/advice-for-public

[3]. Prem, K., et al.: The effect of control strategies to reduce social mixing on outcomes of the covid19 epidemic in Wuhan, China: a modeling study. The Lancet Public Health, (2020) 
[4]. Kahale, N.: On the economic impact of social distancing measures. SSRN Electron. J. (2020). https://doi.org/10.2139/ ssrn.3578415

[5]. Morato, M. M., Bastos, S. B., Cajueiro, D. O. \& NormeyRico, J. E. An optimal predictive control strategy for COVID-19 (SARS-CoV-2) social distancing policies in Brazil. Elsevier Annu. Rev. Control. DOI: https://doi.org/ 10.1016/j.arcontrol.2020.07.001 (2020).

[6]. covid19.who.int. (n.d.). WHO coronavirus disease (COVID-19) dashboard. Available at: https://covid19.who.int [Accessed 1 Jul 2020]

[7]. Kajabad, E.N., Ivanov, S.V.: People detection and finding attractive areas by the use of movement detection analysis and deep learning approach. Procedia Comput. Sci. 156, 327-337 (2019). (ISSN 1877-0509)

[8]. C, R. T. Game theory of social distancing in response to an epidemic. PLoS computational biology 1-9, DOI: 10.1371/journal.pcbi.1000793 (2010).

[9]. World Health Organisation. WHO Coronaviruses Disease Dashboard (August 2020). Available at https:=//covid19.who.int/table.

[10]. WHO Director, Generals. Opening remarks at the media briefing on COVID-19 (2020). WHO generals and directors speeches.

[11]. Organization WH. WHO corona-viruses (COVID-19);. https://www.who.int/healthtopics/coronavirus.

[12]. Vision-Based Social Distancing and Critical Density Detection System for COVID19, Department of Electrical and Computer Engineering, The Ohio State University, Columbus, $\quad \mathrm{OH} \quad 43210, \quad$ USA; yurtsever.2@osu.edu (E.Y.); renganathan.5@osu.edu (V.R.); redmill.1@osu.edu (K.A.R.); ozguner.1@osu.edu (Ü.Ö.) in this explain the intro part of the monitoring vision base social distance
[13]. Monitoring COVID-19 social distancing with person detection and tracking via finetuned YOLO v3 and Deepsort techniques in this paper explain the methods and working implementations

\section{Cite this article as :}

Kishan Ghanshyam Poriya, Prof. Surabhi Thorat, Prof. Swati Maurya,"Social Distance Analysing COVID-19 Using Deep Learning and Computer Vision", International Journal of Scientific Research in Computer Science, Engineering and Information Technology (IJSRCSEIT), ISSN : 2456-3307, Volume 7 Issue 6, pp. 196-202, November-December 2021. Available

doi : https://doi.org/10.32628/CSEIT217646

Journal URL : https://ijsrcseit.com/CSEIT217646 